\title{
Stop Motion no Ensino: Um Relato de Experiência na Formação de Professores Via Ensino Mediado por Tecnologia no Amazonas
}

\author{
Almir de O. Costa Junior'1, Lana Barros de Matos² \\ ${ }^{1}$ Escola Superior de Tecnologia - Universidade do Estado do Amazonas (UEA) \\ 69050-020 - Manaus - AM - Brasil \\ ${ }^{2}$ Secretaria de Estado de Educação - SEDUC-AM - Manaus - AM - Brasil \\ adjunioreuea.edu.br, matoslana4lgmail.com
}

\begin{abstract}
This work reports an experience of using the Stop Motion technique in the training process of 2800 basic education teachers who work in 59 municipalities in the state of Amazonas through Technology Mediated Education. Such experience was developed within the graduate course in digital literacy at the State University of Amazonas using low-cost materials and the Movie Maker software. At the end of the experiment, it was possible to observe satisfactory evidence of students' understanding of the concept, the possibilities of Stop Motion in education, as well as important aspects of the technical and practical feasibility of the strategy used in this experiment.
\end{abstract}

Resumo. Este trabalho relata uma experiência de utilização da técnica de Stop Motion no processo formativo de 2800 professores da educação básica que atuam em 59 municipios do estado do Amazonas por meio do Ensino Mediado por Tecnologia. Tal experiência foi desenvolvida dentro do curso de pós-graduação em letramento digital da Universidade do Estado do Amazonas utilizando materiais de baixo custo e o software Movie Maker. Ao final da experiência, foi possível observar evidências satisfatórias de entendimento dos alunos sobre o conceito, as possibilidades do Stop Motion na educação, assim como aspectos importantes da viabilidade técnica e prática da estratégia utilizada nesta experiência.

\section{Introdução}

A sociedade em que vivemos apresenta a cada dia novas formas de ver, ser e estar no mundo. E nesse contexto, podemos observar que as tecnologias sempre estiveram presentes em todas estas mudanças, pelas quais nossa sociedade passou e passa constantemente. Neste sentido, é possível verificar que os seres humanos têm buscado novos modos, meios e recursos que auxiliem as pessoas a se desenvolverem no planeta e no contexto social em que estão inseridas. Na educação não poderia ser diferente. Uma vez que a cada dia emergem desafios a serem superados e o aprender mais e melhor torna-se um imperativo, as tecnologias da informação e comunicação vem exercer papel preponderante nessa conjuntura.

Portanto, os professores necessitam letrar-se digitalmente, conhecer os mecanismos das tecnologias, a dinâmica dos processos, os recursos existentes que 
podem expandir conhecimentos de seus alunos, nosso público alvo. É nesse panorama que se trabalhou a inserção de recursos tecnológicos no curso de pós-graduação em Letramento Digital.

Nessa continuidade, este trabalho apresenta um relato de experiência da utilização da técnica de Stop Motion em um processo formativo de professores da educação básica que atuam em 59 municípios do estado do Amazonas. Tal experiência, foi realizada como parte das atividades avaliativas da disciplina Tecnologias Digitais e Práticas Pedagógicas na Educação: Conceitos e Aplicação, do curso de Pós-graduação Lato sensu em Letramento Digital, realizado em parceria com a Secretaria de Estado de Educação e Qualidade do Ensino (SEDUC) e a Universidade do Estado do Amazonas (UEA).

Para apresentar estes resultados, o artigo está assim organizado: A contextualização da formação de professores no Amazonas encontra-se apresentada na Seção 2. Os fundamentos do Stop Motion encontram-se descritos na Seção 3. O planejamento e a aplicação da estratégia são descritos na Seção 4. Os resultados e as discussões são apresentados na seção 5 . Por fim, as considerações finais são pontuadas na Seção 6.

\section{A Formação de Professores no Amazonas para uso de Tecnologias Digitais}

O Governo do Estado do Amazonas, através da Secretaria Estadual de Educação do Amazonas - SEDUC/AM vem sistematicamente investindo em equipamentos e tecnologias na perspectiva de melhorar a infraestrutura das escolas. Todavia, a aquisição de equipamentos de forma isolada da formação e especialização dos professores para o usufruto das tecnologias nas práticas pedagógicas, não têm surtido o efeito esperado nas escolas.

Diante deste cenário, a Universidade do Estado do Amazonas - UEA em parceria com a SEDUC/AM, propôs a criação do curso de Pós-Graduação Lato Sensu em "Letramento Digital", na perspectiva de capacitar o maior número de professores, das mais diversas áreas de conhecimento, a utilizar as tecnologias digitais da informação e comunicação em processos educativos.

A proposta de curso de pós-graduação lato sensu foi ofertada em duas modalidades: i - Presencial Mediado por Tecnologia e ii - Presencial. Neste trabalho, iremos relatar apenas uma experiência de aplicação nas turmas da modalidade Presencial Mediada Por Tecnologia, que envolveu 2800 alunos distribuídos em 60 turmas em 59 municípios do estado do Amazonas.

De modo geral, o curso esteve organizado em 11 disciplinas. Contudo, iremos relatar apenas uma experiência desenvolvida dentro da disciplina Tecnologias Digitais e Práticas Pedagógicas na Educação, uma disciplina de natureza prática. As aulas nesta modalidade, foram planejadas e transmitidas via sistema IPTV disponível no Centro de Mídias do Amazonas.

O Centro de Mídias de Educação do Amazonas é uma iniciativa do Governo Estadual que visa ampliar e diversificar o atendimento aos alunos da rede pública de ensino do estado, oferecendo uma educação inovadora, por meio das tecnologias da informação e comunicação, com ênfase na interatividade [CEMEAM 2020] por meio da 
VAT S.A que é uma empresa de Tecnologia da Informação, especializada em elaborar e executar projetos de educação em massa e comunicação corporativa, há 16 anos no mercado, com resultados expressivos e prêmios internacionais. Reúne, de forma integrada, especialistas em educação, telecomunicação, desenvolvimento de software e produção de TV [VAT 2020].

\section{Fundamentos do Stop Motion}

Para que possamos falar da técnica Stop Motion e o leitor possa compreender do que se trata, faz-se necessário conhecer onde surgiu e em que contexto a técnica foi criada. Para tanto, é preciso buscar informações que datam do final do século XIX, precisamente entre os anos de 1895 e 1899. Nesse sentido, sendo reconhecida como uma das mais antigas técnicas de animação, trata-se de uma captura da imagem, frame a frame [Paula, Paula e Henrique 2017].

O ano era 1895 e os irmãos Lumiere, franceses, faziam sua primeira apresentação cinematográfica. Muitos outros simpatizantes da sétima arte também davam seus passos, no entanto os Lumieres possuíam equipamentos que à época os concedia vantagem [Guimarães e Gino 2014]. Nesse contexto de inovação tecnológica daquele momento histórico, é que diversos estudiosos do tema vieram ganhando espaço. O também francês George Meliés, nos anos de 1897 adquire uma máquina semelhante à dos irmãos Lumiere e inicia sua jornada numa técnica que posteriormente seria conhecida como Stop Motion.

Inicialmente, alguns autores afirmam que a técnica havia nascido de um "erro", que, no entanto, transformou-se em algo promissor e diferente das iniciativas visuais desenvolvidas até então. Trata-se de um congelamento de cena que possibilitou uma espécie de ilusão de ótica, que é uma das características do Stop Motion [Paula, Paula e Henrique 2017].

Com o passar do tempo a técnica veio ganhando espaço nas mais diversas produções cinematográficas, propagandas e no contexto educacional. O surgimento de novas tecnologias cada vez mais avançadas, trazem ao mundo cinematográfico alguns desafios. Mesmo com as animações produzidas em alta definição, o Stop Motion coexiste com elas, uma vez que são técnicas diferentes e que possuem em seu bojo finalidades distintas.

Algumas das animações mais famosas, encabeçam a lista de produções realizadas a partir da técnica do Stop Motion, a exemplo do filme "O estranho mundo de Jack" e a "Noiva cadáver" de Tim Burtom (1993 e 2005), Coraline de Neil Gaimam (2002), Morph, Wallace \& Gromit e Shaun the Sheep e o longa-metragem A fuga das galinhas do estúdio inglês Ardman (2004).

Por tratar-se de uma tarefa dinâmica, pressupõe que o projetista em Stop Motion desenvolva algumas habilidades, tais como: autonomia, criatividade, senso de organização. Tais habilidades são extremamente necessárias no contexto educacional, ainda mais se o objetivo das ações educativas for incluir o aluno de maneira ativa no processo de ensino-aprendizagem.

O Stop Motion pode ser inserido na educação como um dos recursos tecnológicos trazidos pela grande demanda das Tecnologias da Informação e Comunicação (TIC), que visam alavancar o ensino de modo a possibilitar um 
multiletramento das mais variadas linguagens existentes na atualidade, permitindo ao aluno um maior contato com linguagens que fazem e farão parte de seu cotidiano [Karwoski e Rojo 2010].

Muitos trabalhos vêm sendo desenvolvidos no cenário educacional como [Freitas, Marinho, Santos e Neto 2016; Nascimento 2016; Martins, Galego e Araújo 2017; Santos e Kondo 2019] visando introduzir a técnica no contexto de sala de aula e formação pedagógica, uma vez que de maneira artesanal possibilita ao aluno um contato direto com materiais concretos, desenvolvendo sua percepção artística, lógica e organizacional.

Além disso, esta técnica prevê que os alunos utilizem Softwares de fácil manuseio como o Pic Pac Stop Motion que é um aplicativo para Smartphone e o Windows Movie Maker um editor de vídeo do próprio Windows, igualmente de fácil utilização. A técnica criada no final do século XIX, foi incrivelmente atualizada, por meio dos aparatos tecnológicos, hoje, existentes. No entanto sua essência permanece inalterada, tendo sua contribuição na educação legitimada por pesquisadores que replicam, colhendo bons frutos.

\section{O Planejamento e Aplicação da Estratégia}

$\mathrm{Na}$ experiência relatada neste artigo, será levada em consideração apenas a $2^{\mathrm{a}}$ aula da disciplina Tecnologias Digitais e Prática Pedagógicas na Educação, ou seja, 10 horas/aula das 60 previstas para esta disciplina. De modo geral, esta disciplina tinha por objetivo principal, apresentar aos alunos do curso de pós-graduação, tecnologias digitais e suas possibilidades dentro do processo de ensino e aprendizagem.

\subsection{Execução da Aula 02 - Período da Manhã}

A $2^{\mathrm{a}}$ aula tinha por finalidade apresentar e discutir as possibilidades de utilização do vídeo como ferramenta para auxiliar o processo de ensino e aprendizagem de conteúdos de disciplinas curriculares. De modo geral, esta aula teve a duração de 10 horas, distribuídas no período da manhã e tarde e foi mediada pelo uso do sistema de IPTV do Centro de Mídias do Amazonas.

No período da manhã, por meio de aula expositiva utilizando cartelas (como são chamados os slides dentro do centro de mídias), foram apresentadas aos alunos algumas indagações, tais como: i - Para quê usar o vídeo na escola? ii - Como o vídeo tem sido utilizado na sala de aula? iii - Como os alunos ficam durante aulas que utilizam exibição de vídeos? e iv - Como utilizar o vídeo em atividades ativas em sala de aula?

Além disso, foram apresentados alguns exemplos de ferramentas pagas e gratuitas que permitem realizar a captação, produção e edição de vídeos. Neste contexto, ao final da manhã foi realizada uma apresentação de alguns dos recursos e ferramentas disponíveis no Software Movie Maker, tais como: i - Importar mídias, ii Adicionar títulos, legendas e créditos, iii - Noções de recorte de áudio e vídeo, iv Aplicação de animações, transições e efeitos, v - Salvando projeto e filme, etc.

A apresentação desta ferramenta se deu de duas formas: i - Foram utilizadas cartelas ilustrando as principais opções e recursos disponíveis no Software (Figura 1a) e ii - Uma demonstração ao vivo de como realizar a edição de um vídeo utilizando o Movie Maker (Figura 1b). Neste último caso, a produtora responsável por toda a parte 
de captação, geração e transmissão das imagens para todos os municípios do interior, compartilharam a tela do computador utilizado no estúdio para que os alunos pudessem visualizar todos os passos realizados na demonstração da ferramenta.

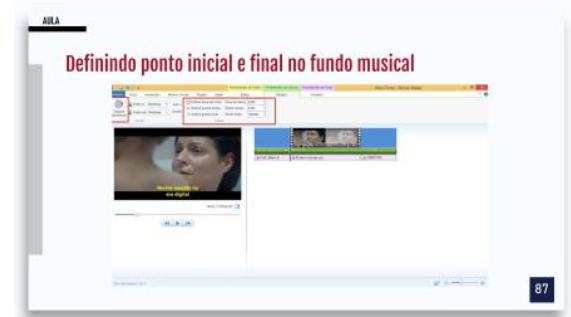

(a) Cartela ilustrando os recursos

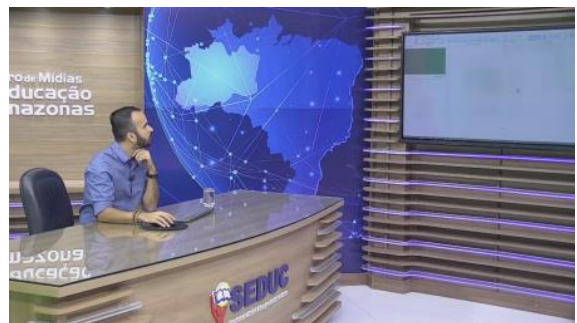

(b) Demonstração da ferramenta

Figura 1. Momentos da apresentação dos recursos e ferramentas do Movie Maker

Para finalizar a parte da manhã da $2^{\mathrm{a}}$ aula, foram realizados mais dois momentos: i - Atividade prática de construção de um curta-metragem e ii - Realização de uma interatividade. Neste último caso, os municípios que solicitaram participar, realizaram uma interação com o professor em estúdio para socializar a atividade desenvolvida por sua equipe em seu município.

\subsection{Execução da Aula 02 - Período da Tarde}

Como foco principal deste artigo, apresentamos nesta seção a organização e aplicação da segunda parte da aula 02 .

Esta parte, tinha por finalidade apresentar e discutir as possibilidades de utilização da técnica do Stop Motion como ferramenta para auxiliar o processo de ensino e aprendizagem de conteúdos de disciplinas curriculares.

\subsubsection{Apresentação do Conceito de Stop Motion}

Em um momento inicial, foram apresentados aos alunos algumas informações a respeito da técnica, tais como: i - O que é Stop Motion?, ii - Conceito de Frame, iii - Como é elaborada uma produção em Stop Motion?, iv - Conceito de Efeitos Especiais, v Exemplos de Filmes produzidos com Stop Motion, vi - Exemplo de Roteiro, vii - Como utilizar o Stop Motion em práticas pedagógicas?, viii - Vantagens de utilizar o Stop Motion em sala de aula, ix - Exemplos de animações produzidas utilizando a técnica em sala de aula, etc. Esta parte da aula, teve a duração de 1 hora. Na Figura 2a é apresentado um exemplo de cartela utilizada para explicar a técnica do Stop Motion e na Figura $2 \mathrm{~b}$ um exemplo de cartela explicando o passo a passo para produzir uma animação em Stop Motion.

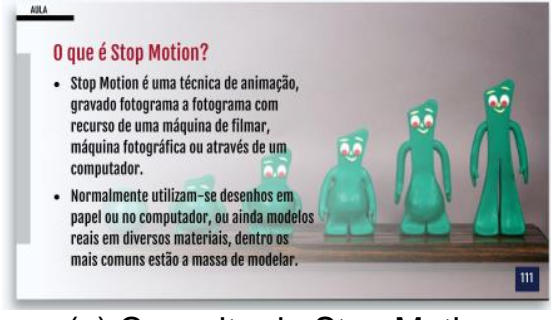

(a) Conceito de Stop Motion

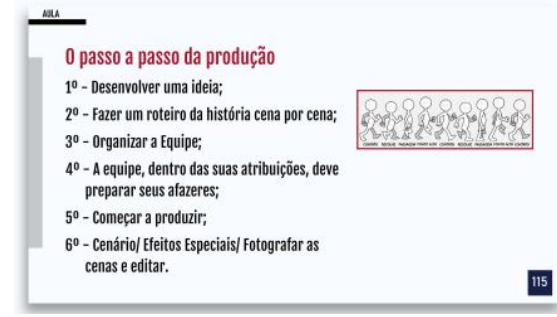

(b) Etapas para produção

Figura 2. Exemplos de cartelas utilizadas para explicar fundamentos do Stop Motion 
Além disso, foram apresentados alguns exemplos de aplicativos (PicPac - Stop Motion) e Softwares (Movie Maker) que podem ser utilizados para desenvolver atividades com esta técnica.

\subsubsection{Apresentação das Ferramentas de Edição}

Dando continuidade à aula, o professor realizou uma demonstração ao vivo no estúdio de como realizar a produção da animação. Nesse sentido, com uma sequência de fotos registradas previamente, o professor demonstrou utilizando o Software Movie Maker a edição de uma animação em Stop Motion.

$\mathrm{Na}$ ocasião, foram demonstradas algumas particularidades sobre $\mathrm{o}$ posicionamento da câmera (Figura 3a), a quantidade de fotos/frames por segundo e a configuração básica sobre o tempo de exibição de uma foto/frame no Software Movie Maker (Figura 3b).

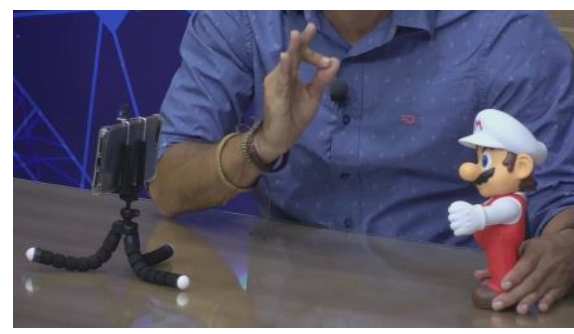

(a) Posicionamento da câmera

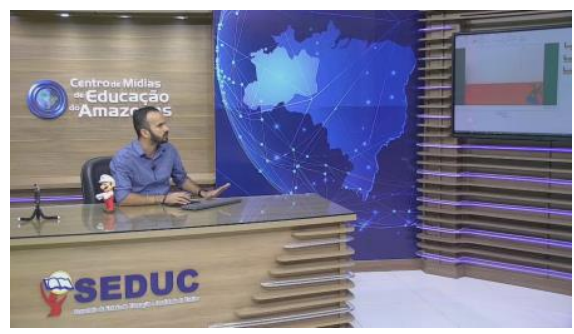

(b) Configurando o tempo do frame

Figura 3. Dicas sobre o processo de captação e produção da animação.

Após realizar a explicação de todos os conceitos, ferramentas e realizar uma demonstração prática de como produzir, foi proposto para os alunos uma atividade prática de construção de uma animação utilizando a técnica do Stop Motion.

\subsubsection{Atividade Prática Utilizando a Técnica do Stop Motion}

De modo geral, a atividade solicitava que os alunos produzissem um vídeo utilizando a técnica do Stop Motion. O vídeo deveria abordar algum conceito de uma disciplina curricular da educação básica (ex. fotossíntese, movimento de rotação e translação da terra, etc.). Além disso, alguns requisitos mínimos para a elaboração do vídeo foram definidos, tais como: i - tempo de duração do vídeo entre 30 a 60 segundos, ii quantidade mínima de 5 frames/fotos por segundo, iii - conter uma abertura, legendas, trilha sonora e créditos e iv - grupos de no máximo 3 alunos.

Para realizar esta atividade, os alunos tinham à disposição os seguintes materiais: i - aparelho celular (com cabo usb), ii - um notebook com o Movie Maker instalado, iii - tubo de cola, iv - tesoura, v - papel A4, vi - papel criative, vii - papel crepom e viii - massa de modelar. Além destes materiais mínimos, foi sugerido que os alunos utilizassem outros materiais alternativos, tais como: i - copo de plástico, ii canudos, iii - palitos de churrasco e picolé etc. A lista dos materiais necessários, foi solicitada no final da $1^{\mathrm{a}}$ aula (sábado anterior a esta aula).

Na perspectiva de uma otimização do tempo disponível para a execução desta atividade, foi disponibilizado um template de roteiro para que os grupos pudessem realizar o planejamento da sua animação. Além disso, um tutorial em .PDF sobre o Software Movie Maker e um roteiro de aprendizagem com uma descrição mais detalhada sobre a atividade, foram elaborados e disponibilizados para os alunos 
utilizando o recurso de compartilhamento de arquivos disponível no sistema de IPTV do centro de mídias do Amazonas.

Em essência, foram disponibilizados aos grupos 1 hora e 30 minutos para realizar esta atividade. Nas Figuras $4 \mathrm{a}, 4 \mathrm{~b}, 4 \mathrm{c}$ e $4 \mathrm{~d}$ pode ser observado os alunos dos municípios desenvolvendo a atividade proposta. As imagens foram compartilhadas por eles via Whatsapp.

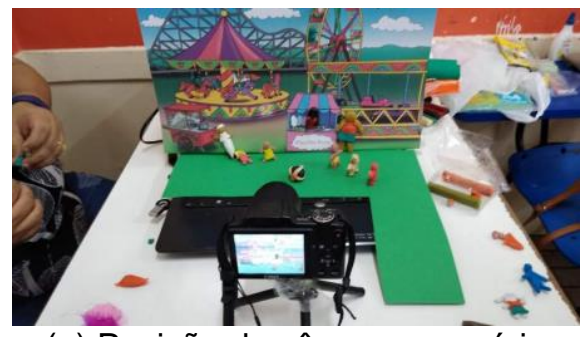

(a) Posição da câmera e cenário

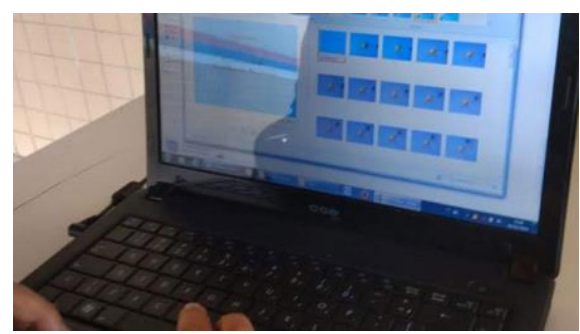

(c) Editando a animação

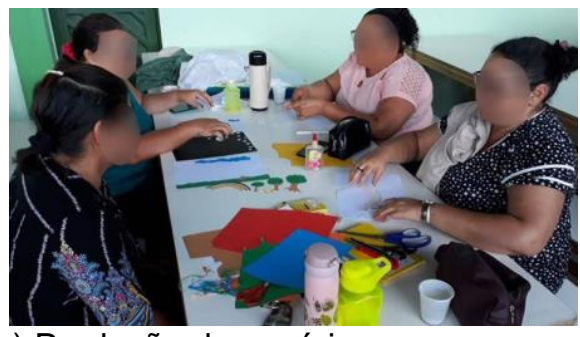

(b) Produção de cenários e personagens

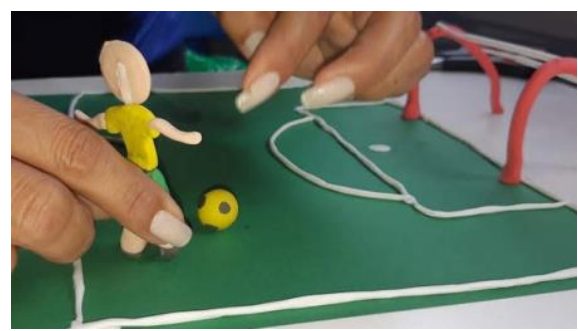

(d) Movimentando os personagens

Figura 4. Alunos desenvolvendo atividade prática com a técnica do Stop Motion.

\subsubsection{Socialização das Atividades - Interatividade}

No momento final desta aula, foi realizada uma interatividade para que alguns municípios pudessem socializar com o professor no estúdio e demais municípios, os vídeos/animações produzidos. Por meio da utilização de uma webcam e microfone, alunos realizaram uma videoconferência onde puderam exibir em seus computadores, o resultado da produção dos vídeos utilizando a técnica do Stop Motion. Diante da grande quantidade de turmas/municípios atingidos pelo programa de pós-graduação, foram realizadas interações apenas com as turmas que solicitaram a vez via aplicativo do IPTV. Esta parte final da aula, teve a duração de 30 minutos.

\section{Resultados e Discussões}

Nesta seção, serão destacados alguns aspectos importantes sobre o processo de condução desta aula, os produtos apresentados e uma avaliação realizada com uma pequena amostragem do total de alunos envolvidos. Inicialmente, com base no relato dos alunos coletados via whatsapp, pôde-se verificar que em essência a grande maioria dos municípios conseguiu finalizar a atividade proposta nesta segunda parte da aula.

Em alguns relatos, foi possível identificar algumas dificuldades em adquirir alguns materiais solicitados para desenvolver a atividade em sala de aula, tais como: Papel criative e massa de modelar. De certo modo, isto se deve ao fato de que algumas cidades estão situadas a mais de $1.000 \mathrm{~km}$ de distância da capital, restringindo o acesso a estes tipos de materiais mais específicos. 
Além disso, é importante destacar que muitos alunos se utilizaram da criatividade para improvisar, por exemplo, um tripé para realizar a captura das imagens (Figuras 5a e 5b).

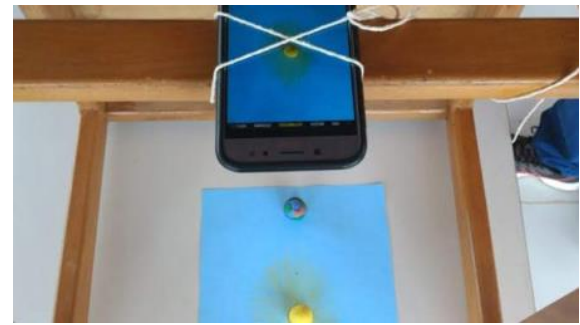

(a) Tripé improvisado com a cadeira

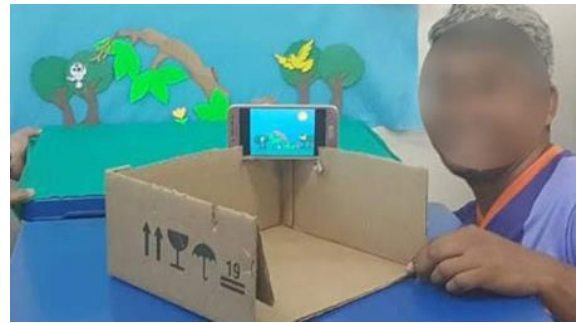

(b) Tripé com caixa de papelão

Figura 5. Alunos improvisando um tripé com materiais alternativos.

De modo geral, os produtos apresentados ao final desta aula, puderam nos fornecer uma análise mais detalhada sobre o impacto ocasionado na formação destes alunos (professores). Nas Figuras 6a e 6b podemos observar dois printscreens do resultado final de alguns dos vídeos produzidos e enviados pelos alunos.

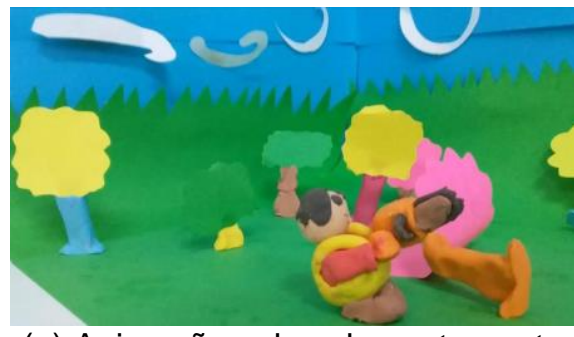

(a) Animação sobre desmatamento

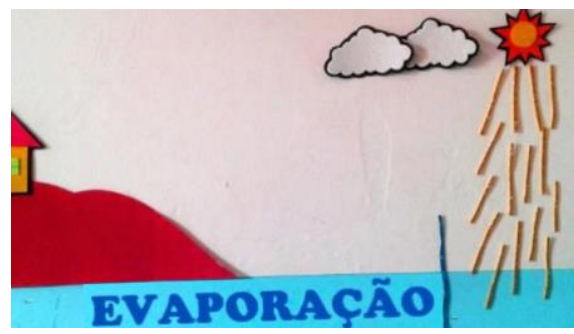

(b) Animação sobre evaporação

Figura 6. Printscreen de algumas animações produzidas pelos alunos.

No total, foram enviados via Google Forms, 188 vídeos abordando temas diferenciados, tais como: i - Educação para o trânsito, ii - Movimentos de rotação e translação da terra, iii - Metamorfose das borboletas, iv - Meio ambiente etc. Por fim, na perspectiva de obter um feedback dos alunos a respeito do objetivo, recursos, ferramentas e atividades desta aula, foi elaborado e enviado aos alunos um questionário do Google Forms. O questionário possuía 9 perguntas e foi respondido por 346 alunos em um universo de 60 turmas (2800 alunos) envolvidas no curso de pós-graduação.

Em 8 questões, os alunos deveriam responder com base em uma escala Likert de 5 níveis: 1 - Discordo Totalmente, 2 - Discordo Parcialmente, 3 - Não Concordo e nem Discordo, 4 - Concordo Parcialmente e 5 - Concordo Totalmente. Em uma das questões (questão 9), os alunos deveriam selecionar dentre as opções preestabelecidas.

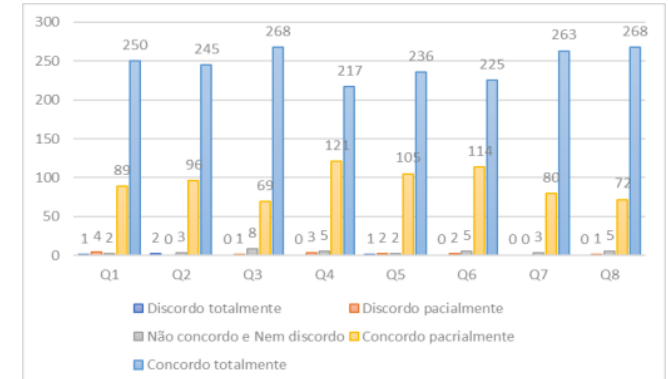

Gráfico 1. Respostas das questões 1, 2, 3, $4,5,6,7$ e 8

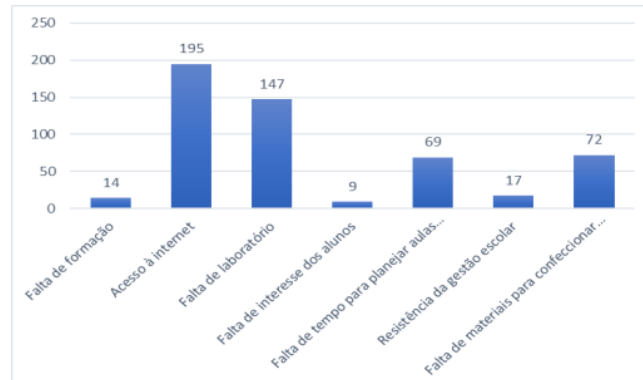

Gráfico 2. Resposta dada pelos alunos na questão 9 
Quando perguntados se foi possível compreender os conceitos, ferramentas e recursos do Stop Motion, através do material, ilustrações e exemplos apresentados na aula, foi possível verificar que $250(72,3 \%)$ alunos afirmaram concordar totalmente (Gráfico 1-Q1).

Em seguida, perguntamos aos alunos se a quantidade de informações e recursos disponibilizados, foram suficientes para compreender os fundamentos da utilização do Stop Motion no Ensino, 245 (70,8\%) dos alunos afirmaram concordar totalmente (Gráfico 1-Q2).

$\mathrm{Na}$ questão 3, os alunos foram indagados se os tutoriais disponibilizados, descrevendo os recursos e explicando o processo de utilização do Movie Maker para trabalhar com Stop Motion ajudaram na realização das atividades, 268 (77,5\%) dos alunos afirmaram concordar totalmente (Gráfico 1 - Q3).

Também perguntamos aos alunos se a partir do que foi ensinado eles estariam se sentindo seguros para utilizar a técnica do Stop Motion como recurso didático tecnológico dentro e fora da sala de aula, 217 (62,7\%) alunos concordaram totalmente (Gráfico 1-Q4).

$\mathrm{Na}$ questão 5, solicitamos que os alunos respondessem se a atividade proposta ao final da aula, permite-lhes compreender todos os conceitos e ferramentas de uma melhor forma, 235 (68,2\%) alunos afirmaram concordar totalmente (Gráfico 1 - Q5).

Em seguida, perguntamos aos alunos se ao utilizarmos o modelo de ensino mediado por tecnologia, foi possível compreender todos os conceitos e ferramentas abordados, $225(65 \%)$ alunos afirmaram concordar totalmente (Gráfico 1 - Q6). Também perguntamos aos alunos se a utilização de roteiros de aprendizagem ajudou a compreender o que deveria ser executado nas atividades, podemos observar que 263 (76\%) alunos afirmaram concordar totalmente (Gráfico 1 - Q7).

Na questão 8 indagamos os alunos se a demonstração da ferramenta e a técnica do Stop Motion ao vivo contribuiu para compreender melhor os conceitos e recursos apresentados na aula, $268(77,5 \%)$ alunos afirmaram concordar totalmente (Gráfico 1 Q8).

Por fim, na questão 9 os alunos foram questionados sobre quais seriam as principais dificuldades para que eles utilizassem a técnica do Stop Motion como recurso didático em sua escola/município. Nesta pergunta, foram preestabelecidas algumas alternativas: i - Falta de formação, ii - Acesso restrito a internet, iii - Ausência de laboratório de informática, iv - Falta de tempo para planejar aulas, $\mathrm{v}$ - Resistência da gestão escolar, e vi - Ausência de materiais para atividade. Foi possível verificar que $195(56,4 \%)$ alunos responderam que a maior dificuldade seria o acesso restrito a internet (Gráfico 2 - Q9).

\section{Considerações Finais}

De modo geral a experiência proporcionou aos alunos/professores um maior estreitamento com os recursos apresentados. Os materiais de apoio como os tutoriais, também foram de grande valia para o entendimento das tarefas e aplicação da técnica sugerida. No entanto, algumas dificuldades como materiais para executar a tarefa, falta de tempo, improvisos - que se mostraram um elemento que possibilitou a criatividade e principalmente a conexão de internet de baixa qualidade oferecida no interior do Estado, 
compromete a atualização dos alunos em relação às novas tecnologias existentes. A partir das produções, foi possível identificar o entendimento por parte dos alunos do que deveria ser realizado e a relação que fizeram entre os conteúdos e a técnica do Stop Motion.

É oportuno dizer, que através das respostas dos alunos no tocante a resistência para o uso de tecnologias, falta de laboratórios de informática e formação em serviço, faz-se necessário repensar sobre, como a evolução tecnológica está sendo recebida por esse público? A necessidade de se criar espaços para o conhecimento, debate e aplicação de novas tecnologias na escola, deve ser estimulado, bem como a participação desses professores deve ser garantida de tal maneira que todos possam ser alcançados, estando o aluno e professor na capital ou no interior do estado.

Como trabalhos futuros, pretende-se realizar uma análise mais detalhada sobre as animações produzidas pelos alunos, na perspectiva de identificar se todos os objetivos/requisitos da atividade solicitada foram atendidos e realizar uma análise crítica das temáticas e sobre a técnica do Stop Motion em cada animação.

\section{Referências}

CEMEAM (2020), Site do Centro de Mídias do Amazonas, https://www.centrodemidias.am.gov.br, Maio.

Freitas, N. A., Marinho, I. W. T., dos Santos, M. N., \& Neto, O. B. (2016). Prática pedagógica com celular e Stop-motion em interdisciplinaridade com Artes. In Anais do Workshop de Informática na Escola (Vol. 22, No. 1, p. 953).

Guimarães, G. B. C., \& Gino, M. S. (2014). Histórico e desenvolvimento do stopmotion e dos personagens articulados no cinema de animação. REVISTA ELETRÔNICA EXTENSÃO EM DEBATE, 1(1).

Karwoski, A. M. (2010). ROJO, Roxane. Letramentos múltiplos, escola e inclusão social. São Paulo: Parábola Editorial, 2009. 128 p. DELTA: Documentação e Estudos em Linguística Teórica e Aplicada, 26(1).

Nascimento, J. M. Stop Motion como estratégia metodológica aplicada ao ensino de Biologia: Relato de experiência didática no âmbito do PIBID. 28f. Trabalho de Conclusão de Curso (Graduação em Ciências Biológicas) - Universidade Estadual da Paraíba, Campina Grande.

Martins, G, Galego, L. G. C., Araujo, C. H. M., Análise da produção de vídeos didáticos de Biologia Celular em stop motion com base na Teoria Cognitiva de Aprendizagem Multimídia. Revista Brasileira de Ensino de CiÊncia e Tecnologia, Ponta Grossa, v. 10, n. 3, p.185-205.

Paula, J. L., Paula, J. L., \& Henrique, A. L. S. (2017). O uso do stop-motion como prática pedagógica no ensino de geografia no contexto do EMI. HOLOS, 3, 141-149.

Santos, S. K., \& Kondo, C. (2019). Stop motion como estratégia de ensino e aprendizagem para crianças, jovens e adultos. In Anais do Workshop de Informática na Escola (Vol. 25, No. 1, p. 1044).

VAT (2020), Site da VAT, https://www.vat.com.br/grupo, Maio. 